\title{
ENVIRONMENTAL EFFECTS ON RF CAVITY TUNING*
}

\author{
J. Sebek ${ }^{\dagger}$ and C. Limborg \\ Stanford Linear Accelerator Center, Stanford, California 94025
}

\begin{abstract}
The SPEAR 2 radio frequency (RF) system uses cavities with minimal external damping, no circulators, and long uncovered waveguide runs. Because of these factors, the strengths and frequencies of the RF system impedances are dependent on such environmental factors as temperature and pressure. These effects are quantified; system modifications to control these effects and therefore improve beam stability are discussed.
\end{abstract}

\section{INTRODUCTION}

The effect of resonator impedances on beam instabilities is well known. In most discussions, these impedances are calculated solely from the RF cavity. The cavity is only one, although by far the most dominant, part of the impedance that the beam sees. In RF systems with long waveguide runs between the cavity and klystron, the details of the waveguide dimensions may have significant effects on the impedance seen by the beam and therefore the resulting beam instability thresholds.

SPEAR is a $3 \mathrm{GeV}$ electron storage ring used for synchrotron radiation. It has two RF stations, each consisting of a $358.533 \mathrm{MHz}$ klystron, waveguide, and cavity. It was built before the various impedance mechanisms causing instabilities were well understood, so its RF accelerating system lacks many of the items now standard in such systems. The RF stations were built with no circulators, temperature regulation, or higher order mode (HOM) dampers. The waveguide runs are long (one runs for 75 meters and the other about half that length) and most of the waveguide is outside, in the open air. Changes in environmental conditions, ambient temperature and pressure, have caused beam instabilities. Fortunately, the thresholds of these observed instabilities have been high enough that, as these causes have been discovered, they have been eliminated with inexpensive solutions targeted for the specific problem.

\section{CAVITY TUNING}

SPEAR long had problems with longitudinal beam instabilities induced by higher order modes (HOM) in the RF cavities. A detailed program to characterize the frequency, strength, and location of these modes (in the operational space of the cavities) was implemented [1]. Careful adjustment of the cavity volume by both setting the position of the adjustable tuners and changing the cavity temperature (indirectly from the strength of the applied accelerating

\footnotetext{
*Work supported by Department of Energy contract DE-AC0376SF00515.

$\dagger$ sebek@slac.stanford.edu
}

voltage) allowed SPEAR to avoid these resonance locations and resulting instabilities during operation.

\section{TRANSVERSE INSTABILITIES}

\subsection{Observations}

After the beam had been stabilized longitudinally, an intermittent transverse instability was observed during the spring and summer. The characteristics of this instability were unexpected in that the instability was strongly correlated with the external ambient temperature. The onset of the instability tracked the morning temperature increase with the rising sun, even though the temperature of the cavity itself, insulated by the SPEAR tunnel shielding, did not change during this time period. Experiments determined that the beam could be stabilized by cooling the external waveguide. Measurements of spectra in the waveguide showed the destabilizing resonance to be located around $1.2 \mathrm{GHz}$.

\subsection{Analysis}

Once the waveguide was found to be a major contributing cause to the instability, it was clear that one could no longer consider the guide to be just a conduit for power flow. It is now an important part of an integrated system in which it connects two resonant cavities, one of which is the accelerating cavity and the other is the klystron.

The impedance seen by the beam is the parallel combination of the cavity impedance with the transformed klystron impedance, where the waveguide acts as the impedance transformer. Using standard transmission line theory, an ideal lossless transmission line, with guide wavenumber $\beta_{g}$ and characteristic impedance $Z_{0}$, transforms a load of impedance $Z_{K}$, at a distance $-d$ from the load, into

$$
Z(-d)=Z_{0} \frac{Z_{K}+i Z_{0} \tan \beta_{g} d}{Z_{0}+i Z_{K} \tan \beta_{g} d}
$$

When the system is matched, i.e., $Z_{K}=Z_{0}$, the guide is transparent, independent of its length, and the beam sees just the parallel combination of the cavity and klystron resonator impedances. As long as the system is nearly matched, slight changes of the guide parameters have no major effect on the system.

At frequencies sufficiently far from the RF frequency, $f_{R F}$, the system is no longer well matched. At frequencies much higher than $f_{R F}$ the klystron impedance $Z_{K}$ is very far above its resonance and thus appears capacitive; $\left|Z_{K}\right| \ll Z_{0}$ and

$$
Z(-d) \simeq i Z_{0} \tan \beta_{g} d .
$$


Now changes to the guide wavenumber, or to the length of guide itself can have dramatic effects on the impedance seen by the beam. (The finite losses in the guide prevent reaching an actual singularity, but the value can still be quite large.)

For safety reasons, the SPEAR waveguides are filled with dry air at a small positive pressure in order to be able to detect physical breaks in the waveguide. Temperature affects these guides in two ways. The aluminum expands and contracts with temperature. But there is a more important effect. The change of temperature changes the guide pressure through the gas law $P V=N k T$. The resulting change in pressure changes the transverse guide dimensions. Increasing pressure causes the rectangular duct to bow out on the broad sides (top and bottom) and the stiff right angle corners force the narrow sides to bow in (figure 1). This change in cross section changes the guide's transverse wavenumber, $k_{\perp}$, which, in turn, changes the longitudinal wavenumber, $\beta_{g}=\sqrt{(\omega / c)^{2}-k_{\perp}^{2}}$.

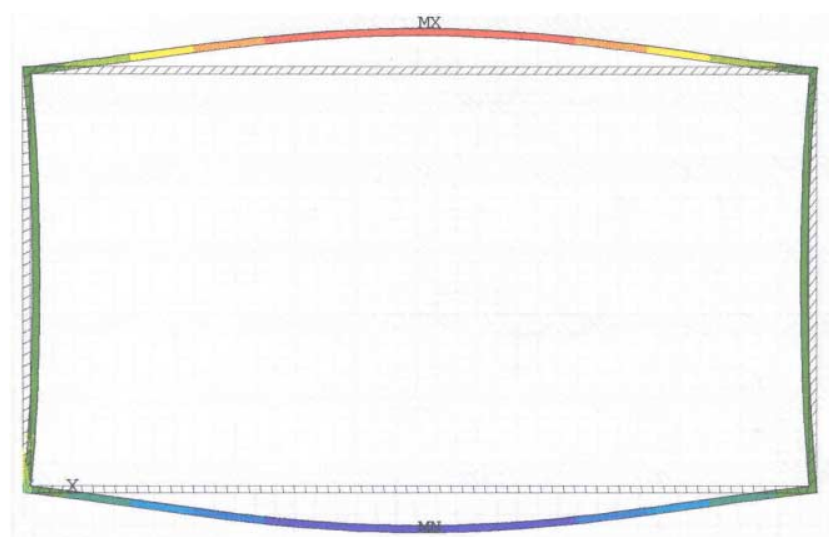

Figure 1: ANSYS output of waveguide deflection under pressure. The constraint of stiff corners causes the short walls to bow in.

The changes in temperature, and the resulting change in pressure, pulled the waveguide impedance through a maximum at a betatron sideband, leading to the transverse instability.

\subsection{Solution}

The guide pressure was unregulated; the average input airflow was just set to equal the average flow out of the controlled leak. The time constant of the system was tens of minutes. The transient caused by a rapid temperature change swept the HOM through the betatron frequency. The solution for this problem was straightforward. The guide pressure was regulated with a flow valve to track the ambient pressure and the instability was cured until last year. Then, during the hottest months of the summer, the instability returned because the limited dynamic range of the pressure control was insufficient to handle the pressure deviations due to large temperature excursions. A set of
HOM dampers, installed on the waveguides last year, has eliminated the instabilities throughout this run [2].

\section{FUNDAMENTAL MODE}

\subsection{Observations}

Although SPEAR has two RF systems, it only uses one in normal operation; the other remains idle. During the last downtime, civil construction in preparation for SPEAR 3 caused the length of the 75 meter waveguide of the normally idle system to be increased by $10 \mathrm{~cm}$ $\left(\beta_{g}=1.35 \mathrm{~m}\right)$. This system behaved as expected when powered up for commissioning purposes in startup, but its behavior changed when it was used as an idle cavity.

The impact on operational performance was noticed in the cold months of the winter. SPEAR's normal daily fill occurs at 06:00. During the cold mornings, a strong longitudinal instability would occur during the fill, enough to cause beam loss and stop the fill. Experimental observations showed that the fundamental mode, $f_{R F}$, was causing the instability. During the instability onset, the power in the fundamental mode and the guide pressure increased rapidly. Applying DC power to the cavity's klystron, while leaving the RF off, stabilized the beam. Although SPEAR could operate stably with this klystron powered, the "energy crisis" in California increased the importance of minimizing the total site energy requirements by running with this klystron off.

\subsection{Analysis}

Armed with our understanding of its importance, we investigated the transformed klystron impedance. As noted above, the SPEAR RF system has no circulators. Therefore power at $f_{R F}$ radiated out of the idle cavity sees the transformed klystron impedance. The klystron is composed of several cavities, each slightly stagger tuned to increase the total bandwidth over that of a single cavity. The last cell was found to be tuned slightly above $f_{R F}$, so the unpowered klystron appears very inductive and mismatched to the guide at that frequency. The $10 \mathrm{~cm}$ change in guide length had moved the phase in the impedance given by Eq. 2 to an odd integral multiple of $\pi / 2$.

Turning on the DC klystron beam has the effect of adding a shunt resistance to the klystron cavity. This change in net impedance is sufficient to reduce the waveguide - klystron mismatch to a level at which the transformed impedance at $f_{R F}$ remains below the instability threshold (figure 2).

This instability is a case of a positive feedback system in which the system starts out with stable beam, but as the beam current increases, both the strength and frequency of the destabilizing resonance change to drive the beam increasingly unstable. Both temperature and pressure changes contribute to the positive feedback.

At "daytime" temperatures, the waveguide dimensions (at the operating point of the waveguide pressure) were 


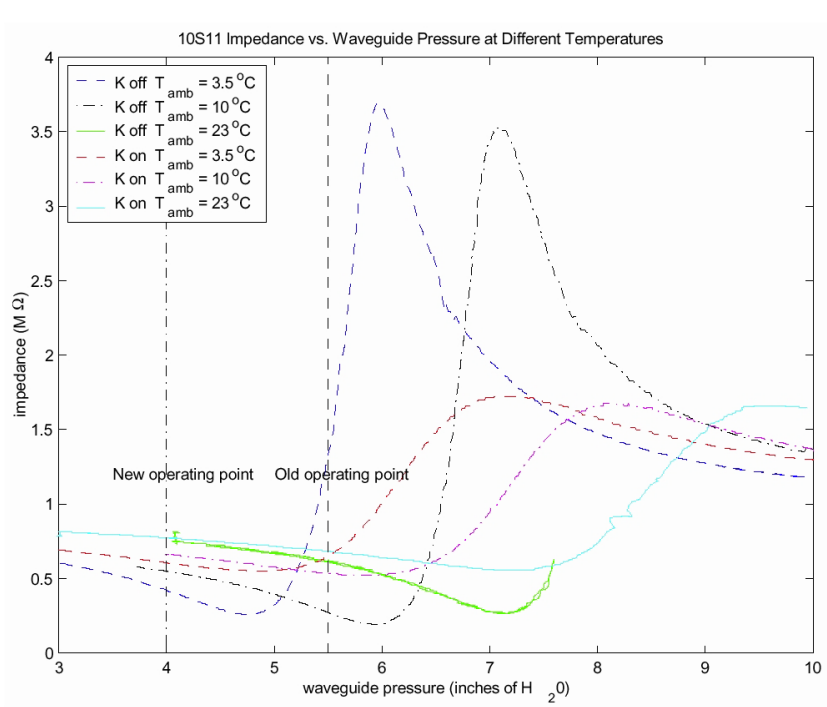

Figure 2: Fundamental resonance plotted vs waveguide pressure for three different temperatures with the klystron on and off.

such that the $\tan \beta_{g} d$ term never became very large. Even though the klystron was still mismatched to the guide, the transformed impedance remained small. At the lower early morning temperatures, however, the dimensional change due to temperature did two things. First, it increased the impedance to a level that approached the instability threshold. Second, it placed the "zero current" value of the impedance on the positive slope of the $Z$ vs $P(T)$ curve.

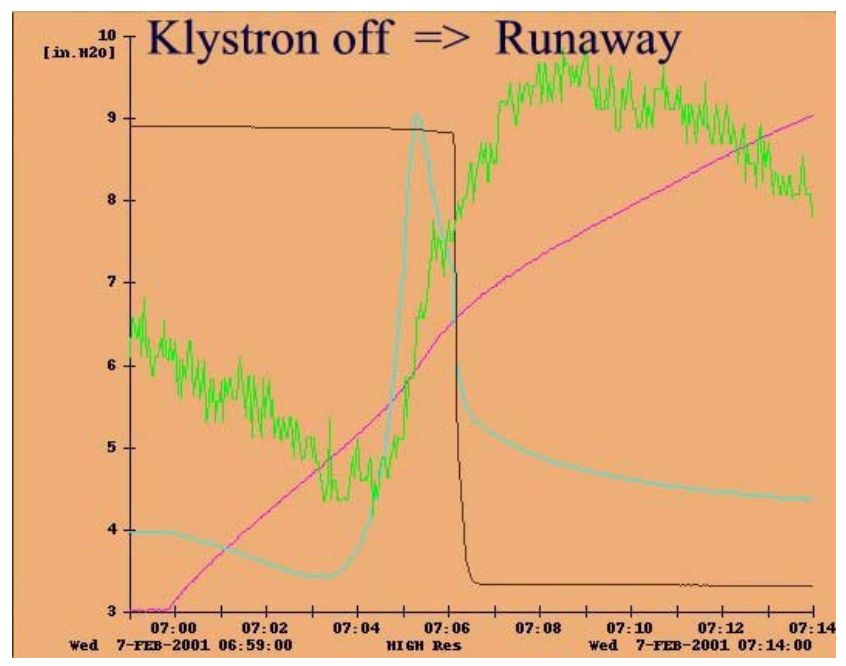

Figure 3: An experimental demonstration of the instability mechanism. The waveguide pressure is allowed to increase smoothly. The monotonically rising curve is the waveguide pressure, induced $V_{g a p}$ is the peaked curve, the jagged curve is the guide temperature and the negative step is the beam current. Note the increase in pressure slope when $V_{\text {gap }}$ starts to peak.
The positive feedback mechanism of this instability can be understood as follows. The fill starts with $Z$ on the positive slope of the $Z$ vs $P(T)$ curve. As the beam current, $I_{b}$, increases, the induced fields in the cavity increase, thereby increasing the standing waves in the waveguide. These fields induce wall currents, $I_{g}$, in the waveguides. The guides are excellent, but not perfect, conductors, so the wall currents cause resistive heating in the walls. This heating increases over time scales that are faster than the time constant of the pressure feedback system, so at these time scales the pressure is again unregulated. $P(T)$ increases and, since $d Z / d P$ is positive, the strength of $Z$ grows. Increasing $Z$, for fixed $I_{b}$, increases the guide fields and therefore $I_{g}$. This is the positive feedback cycle. Figure 3 shows the results of a controlled experiment in which the guide pressure was increased to demonstrate this effect.

\subsection{Solution}

The solution to this problem was straightforward. The waveguide pressure setpoint was changed so that, over the full range of SPEAR ambient temperatures, $\tan \beta_{g} d$ never becomes large. Now at all but the lowest temperatures, $\tan \beta_{g} d$ is negligible (figure 2). And in the range of the lowest ambient temperatures, where $\tan \beta_{g} d$ is noticeable, the operating point is on a portion of the $d Z / d P$ curve that is negative, giving a natural negative feedback condition to guard against against instabilities of this kind.

\section{SUMMARY}

Changes in the ambient temperature and pressure seen by the RF waveguide can cause substantial changes in the impedance seen by the beam, leading to instabilities. By recognizing that the RF system seen by the beam is a two resonator system coupled by a waveguide, one can understand the effects of these environmental effects on the beam. With such an analysis, one recognizes the importance of controlling the environment around the guide, as well as the benefit of the now standard elements of an RF system such as circulators and waveguide HOM dampers.

\section{ACKNOWLEDGEMENTS}

We would like to thank the SSRL accelerator operations staff for their assistance in uncovering the causes of these instabilities. We also thank Max Cornacchia for his strong support in all phases of this accelerator improvement project.

\section{REFERENCES}

[1] C. Limborg and J. Sebek, "Measurement of High Q RF Cavity Impedance with Beam”, EPAC'98, Stockholm, June 1998, p. 972.

[2] J. Sebek, et. al., "SPEAR 2 RF System Loads", PAC'01, Chicago, June 2001. 